\title{
On the new approach to variable separation in the time-dependent Schrödinger equation with two space dimensions
}

\author{
R.Z. Zhdanov \\ Arnold-Sommerfeld Institute for Mathematical Physics, \\ Leibnitzstraße 10, 38678 Clausthal-Zellerfeld, Germany* \\ I.V. Revenko and W.I. Fushchych \\ Institute of Mathematics of the Academy of Sciences of Ukraine, \\ Tereshchenkivska Str.3, 252004 Kiev, Ukraine
}

October 27, 2018

\begin{abstract}
We suggest an effective approach to separation of variables in the Schrödinger equation with two space variables. Using it we classify inequivalent potentials $V\left(x_{1}, x_{2}\right)$ such that the corresponding Schrödinger equations admit separation of variables. Besides that, we carry out separation of variables in the Schrödinger equation with the anisotropic harmonic oscillator potential $V=k_{1} x_{1}^{2}+k_{2} x_{2}^{2}$ and obtain a complete list of coordinate systems providing its separability. Most of these coordinate systems depend essentially on the form of the potential and do not provide separation of variables in the free Schrödinger equation $(V=0)$.
\end{abstract}

\section{Introduction}

The problem of separation of variables $(S V)$ in the two-dimensional Schrödinger equation

$$
\imath u_{t}+u_{x_{1} x_{1}}+u_{x_{2} x_{2}}=V\left(x_{1}, x_{2}\right) u
$$

as well as the most of classical problems of mathematical physics can be formulated in a very simple way (but this simplicity does not, of course, imply an existence of easy

\footnotetext{
* On leave from the Institute of Mathematics of the Academy of Sciences of Ukraine, Tereshchenkivska Str.3, 252004 Kiev, Ukraine

e-mail: asrz@pta3.pt.tu-clausthal.de
} 
way to its solution). To separate variables in Eq.(11) one has to construct such functions

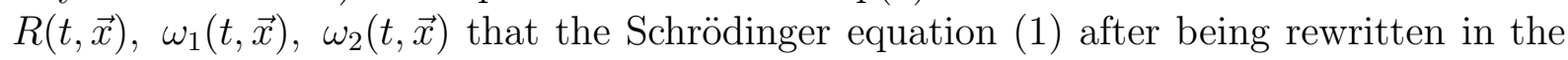
new variables

$$
\begin{aligned}
& z_{0}=t, z_{1}=\omega_{1}(t, \vec{x}), z_{2}=\omega_{2}(t, \vec{x}), \\
& v\left(z_{0}, \vec{z}\right)=R(t, \vec{x}) u(t, \vec{x})
\end{aligned}
$$

separates into three ordinary differential equations $(O D E s)$. From this point of view the problem of $S V$ in Eq.(四) is studied in [1]- 田.

But no less important problem is the one of description of potentials $V\left(x_{1}, x_{2}\right)$ such that the Schrödinger equation admits variable separation. That is why saying about $S V$ in Eq.(1) we imply two mutually connected problems. The first one is to describe all such functions $V\left(x_{1}, x_{2}\right)$ that the corresponding Schrödinger equation (1) can be separated into three $O D E s$ in some coordinate system of the form (2) (classification problem). The second problem is to construct for each function $V\left(x_{1}, x_{2}\right)$ obtained in this way all coordinate systems (2) enabling us to carry out $S V$ in Eq.(11).

Up to our knowledge, the second problem has been solved provided $V=0$ [2, 3] and $V=\alpha x_{1}^{-2}+\beta x_{2}^{-2}[1]$. The first one was considered in a restricted sense in [4]. Authors using symmetry approach to classification problem obtained some potentials providing separability of Eq.(11) and carried out $S V$ in the corresponding Schrödinger equation. But their results are far from being complete and systematic. The necessary and sufficient conditions imposed on the potential $V\left(x_{1}, x_{2}\right)$ by the requirement that the Schrödinger equation admits symmetry operators of an arbitrary order are obtained in [5]. But so far there is no systematic and exhaustive description of potentials $V\left(x_{1}, x_{2}\right)$ providing $S V$ in Eq.(1).

To have a right to say about description of all potentials and all coordinate systems making it possible to separate the Schrödinger equation one has to give a definition of $S V$. One of the possible definitions of $S V$ in partial differential equations ( $P D E s)$ is proposed in our paper [6]. It is based on the concept of Ansatz suggested by Fushchych [7] and on ideas contained in the paper by Koornwinder [8]. The said definition is quite algorithmic in the sense that it contains a regular algorithm of variable separation in partial differential equations which can be easily adapted to handle both linear [6, 9] and nonlinear [10] $P D E s$. In the present paper we apply the said algorithm to solve the problem of $S V$ in Eq.(11).

Consider the following system of ODEs:

$$
\begin{aligned}
\imath \frac{d \varphi_{0}}{d t} & =U_{0}\left(t, \varphi_{0} ; \lambda_{1}, \lambda_{2}\right), \\
\frac{d^{2} \varphi_{1}}{d \omega_{1}^{2}} & =U_{1}\left(\omega_{1}, \varphi_{1}, \frac{d \varphi_{1}}{d \omega_{1}} ; \lambda_{1}, \lambda_{2}\right), \\
\frac{d^{2} \varphi_{2}}{d \omega_{2}^{2}} & =U_{2}\left(\omega_{2}, \varphi_{2}, \frac{d \varphi_{2}}{d \omega_{2}} ; \lambda_{1}, \lambda_{2}\right),
\end{aligned}
$$


where $U_{0}, U_{1}, U_{2}$ are some smooth functions of the corresponding arguments, $\lambda_{1}, \lambda_{2} \subset R^{1}$ are arbitrary parameters (separation constants) and what is more

$$
\operatorname{rank}\left\|\frac{\partial U_{\mu}}{\partial \lambda_{a}}\right\|_{\mu=0 a=1}^{2}=2
$$

(the last condition ensures essential dependence of the corresponding solution with separated variables on $\lambda_{1}, \lambda_{2}$, see 8$]$ ).

Definition 1. We say that Eq.(1) admits $S V$ in the system of coordinates $t, \omega_{1}(t, \vec{x})$, $\omega_{2}(t, \vec{x})$ if substitution of the Ansatz

$$
u=Q(t, \vec{x}) \varphi_{0}(t) \varphi_{1}\left(\omega_{1}(t, \vec{x})\right) \varphi_{2}\left(\omega_{2}(t, \vec{x})\right)
$$

into (1) with subsequent exclusion of the derivatives $\frac{d \varphi_{0}}{d t}, \frac{d^{2} \varphi_{1}}{d \omega_{1}^{2}}, \frac{d^{2} \varphi_{2}}{d \omega_{2}^{2}}$ according to equations (3) yields an identity with respect to $\varphi_{0}, \varphi_{1}, \varphi_{2}, \frac{d \varphi_{1}}{d \omega_{1}}, \frac{d \varphi_{2}}{d \omega_{2}}, \lambda_{1}, \lambda_{2}$.

Thus, according to the above definition to separate variables in Eq.(1) one has

- to substitute the expression (5) into (11),

- to exclude derivatives $\frac{d \varphi_{0}}{d t}, \frac{d^{2} \varphi_{1}}{d \omega_{1}^{2}}, \frac{d^{2} \varphi_{2}}{d \omega_{2}^{2}}$ with the help of equations (3),

- to split the obtained equality with respect to the variables $\varphi_{0}, \varphi_{1}, \varphi_{2}, \frac{d \varphi_{1}}{d \omega_{1}}, \frac{d \varphi_{2}}{d \omega_{2}}, \lambda_{1}$, $\lambda_{2}$ considered as independent.

As a result one gets some over-determined system of PDEs for the functions $Q(t, \vec{x})$, $\omega_{1}(t, \vec{x}), \quad \omega_{2}(t, \vec{x})$. On solving it one obtains a complete description of all coordinate systems and potentials providing $S V$ in the Schrödinger equation. Naturally, an expression complete description makes sense only within the framework of our definition. So if one uses a more general definition it may be possible to construct new coordinate systems and potentials providing separability of Eq.(1). But all solutions of the Schrödinger equation with separated variables known to us fit into the scheme suggested by us and can be obtained in the above described way.

\section{Classification of potentials $V\left(x_{1}, x_{2}\right)$.}

We do not adduce in full detail computations needed because they are very cumbersome. We shall restrict ourselves to pointing out main steps of the realization of the above suggested algorithm.

First of all we make a remark, which makes life a little bit easier. It is readily seen that a substitution of the form

$$
\begin{aligned}
Q & \rightarrow Q^{\prime}=Q \Psi_{1}\left(\omega_{1}\right) \Psi_{2}\left(\omega_{2}\right) \\
\omega_{a} & \rightarrow \omega_{a}^{\prime}=\Omega_{a}\left(\omega_{a}\right), a=1,2, \\
\lambda_{a} & \rightarrow \lambda_{a}^{\prime}=\Lambda_{a}\left(\lambda_{1}, \lambda_{2}\right), a=1,2,
\end{aligned}
$$


does not alter the structure of relations (3), (14), (5). That is why, we can introduce the following equivalence relation:

$$
\left(\omega_{1}, \omega_{2}, Q\right) \sim\left(\omega_{1}^{\prime}, \omega_{2}^{\prime}, Q^{\prime}\right)
$$

provided (6) holds with some $\Psi_{a}, \Omega_{a}, \Lambda_{a}$.

Substituting (5) into (1) and excluding the derivatives $\frac{d \varphi_{0}}{d t}, \frac{d^{2} \varphi_{1}}{d \omega_{1}^{2}}, \frac{d^{2} \varphi_{2}}{d \omega_{2}^{2}}$ with the use of equations (3) we get

$$
\begin{aligned}
& \imath\left(Q_{t} \varphi_{0} \varphi_{1} \varphi_{2}+Q U_{0} \varphi_{1} \varphi_{2}+Q \omega_{1 t} \varphi_{0} \dot{\varphi}_{1} \varphi_{2}+Q \omega_{2 t} \varphi_{0} \varphi_{1} \dot{\varphi}_{2}\right)+(\triangle Q) \varphi_{0} \varphi_{1} \varphi_{2} \\
& \quad+2 Q_{x_{a}} \omega_{1 x_{a}} \varphi_{0} \dot{\varphi}_{1} \varphi_{2}+2 Q_{x_{a}} \omega_{2 x_{a}} \varphi_{0} \varphi_{1} \dot{\varphi}_{2}+Q\left(\left(\triangle \omega_{1}\right) \varphi_{0} \dot{\varphi}_{1} \varphi_{2}+\left(\triangle \omega_{2}\right) \varphi_{0} \varphi_{1} \dot{\varphi}_{2}\right. \\
& \left.\quad+\omega_{1 x_{a}} \omega_{1 x_{a}} \varphi_{0} U_{1} \varphi_{2}+\omega_{2 x_{a}} \omega_{2 x_{a}} \varphi_{0} \varphi_{1} U_{2}+2 \omega_{1 x_{a}} \omega_{2 x_{a}} \varphi_{0} \dot{\varphi}_{1} \dot{\varphi}_{2}\right)=V Q \varphi_{0} \varphi_{1} \varphi_{2},
\end{aligned}
$$

where the summation over the repeated index $a$ from 1 to 2 is understood. Hereafter an overdot means differentiation with respect to a corresponding argument and $\triangle=\partial_{x_{1}}^{2}+\partial_{x_{2}}^{2}$.

Splitting the equality obtained with respect to independent variables $\varphi_{1}, \varphi_{2}, \frac{d \varphi_{1}}{d \omega_{1}}, \frac{d \varphi_{2}}{d \omega_{2}}$, $\lambda_{1}, \lambda_{2}$ we conclude that $O D E s$ (3) are linear and up to the equivalence relation (6) can be written in the form

$$
\begin{aligned}
\imath \frac{d \varphi_{0}}{d t} & =\left(\lambda_{1} R_{1}(t)+\lambda_{2} R_{2}(t)+R_{0}(t)\right) \varphi_{0}, \\
\frac{d^{2} \varphi_{1}}{d \omega_{1}^{2}} & =\left(\lambda_{1} B_{11}\left(\omega_{1}\right)+\lambda_{2} B_{12}\left(\omega_{1}\right)+B_{01}\left(\omega_{1}\right)\right) \varphi_{1} \\
\frac{d^{2} \varphi_{2}}{d \omega_{2}^{2}} & =\left(\lambda_{1} B_{21}\left(\omega_{2}\right)+\lambda_{2} B_{22}\left(\omega_{2}\right)+B_{02}\left(\omega_{2}\right)\right) \varphi_{2}
\end{aligned}
$$

and what is more, functions $\omega_{1}, \omega_{2}, Q$ satisfy an over-determined system of nonlinear PDEs

1. $\omega_{1 x_{b}} \omega_{2 x_{b}}=0$,

2. $B_{1 a}\left(\omega_{1}\right) \omega_{1 x_{b}} \omega_{1 x_{b}}+B_{2 a}\left(\omega_{2}\right) \omega_{2 x_{b}} \omega_{2 x_{b}}+R_{a}(t)=0, \quad a=1,2$,

3. $2 \omega_{a x_{b}} Q_{x_{b}}+Q\left(\iota \omega_{a t}+\triangle \omega_{a}\right), \quad a=1,2$,

4. $\left(B_{01}\left(\omega_{1}\right) \omega_{1 x_{b}} \omega_{1 x_{b}}+B_{02}\left(\omega_{1}\right) \omega_{2 x_{b}} \omega_{2 x_{b}}\right) Q+\imath Q_{t}+\triangle Q+R_{0}(t) Q$ $-V\left(x_{1}, x_{2}\right) Q=0$.

Thus, to solve the problem of $S V$ for the linear Schrödinger equation it is necessary to construct general solution of system of nonlinear PDEs (7). Roughly speaking, to solve a linear equation one has to solve a system of nonlinear equations! This is the reason why 
so far there is no complete description of all coordinate systems providing separability of the four-dimensional wave equation [3].

But in the case involved we have succeeded in integrating system of nonlinear PDEs (7). Our approach to integration of it is based on the following change of variables (hodograph transformation)

$$
\begin{aligned}
& z_{0}=t, z_{1}=Z_{1}\left(t, \omega_{1}, \omega_{2}\right), z_{2}=Z_{2}\left(t, \omega_{1}, \omega_{2}\right), \\
& v_{1}=x_{1}, v_{2}=x_{2}
\end{aligned}
$$

where $z_{0}, z_{1}, z_{2}$ are new independent and $v_{1}, v_{2}$ are new dependent variables correspondingly.

Using the hodograph transformation determined above we have constructed the general solution of equations 1-3 from (7). It is given up to the equivalence relation (6) by one of the following formulae:

1. $\omega_{1}=A(t) x_{1}+W_{1}(t), \quad \omega_{2}=B(t) x_{2}+W_{2}(t)$,

$Q(t, \vec{x})=\exp \left\{-\frac{\imath}{4}\left(\frac{\dot{A}}{A} x_{1}^{2}+\frac{\dot{B}}{B} x_{2}^{2}\right)-\frac{\imath}{2}\left(\frac{\dot{W}_{1}}{A} x_{1}+\frac{\dot{W}_{2}}{B} x_{2}\right)\right\}$

2. $\omega_{1}=\frac{1}{2} \ln \left(x_{1}^{2}+x_{2}^{2}\right)+W(t), \quad \omega_{2}=\arctan \frac{x_{1}}{x_{2}}$,

$$
Q(t, \vec{x})=\exp \left\{-\frac{\imath \dot{W}}{4}\left(x_{1}^{2}+x_{2}^{2}\right)\right\}
$$

3. $x_{1}=\frac{1}{2} W(t)\left(\omega_{1}^{2}-\omega_{2}^{2}\right)+W_{1}(t), \quad x_{2}=W(t) \omega_{1} \omega_{2}+W_{2}(t)$,

$$
Q(t, \vec{x})=\exp \left\{\frac{\imath \dot{W}}{4 W}\left(\left(x_{1}-W_{1}\right)^{2}+\left(x_{2}-W_{2}\right)^{2}\right)+\frac{\imath}{2}\left(\dot{W}_{1} x_{1}+\dot{W}_{2} x_{2}\right)\right\}
$$

4. $x_{1}=W(t) \cosh \omega_{1} \cos \omega_{2}+W_{1}(t), \quad x_{2}=W(t) \sinh \omega_{1} \sin \omega_{2}+W_{2}(t)$,

$$
Q(t, \vec{x})=\exp \left\{\frac{\imath \dot{W}}{4 W}\left(\left(x_{1}-W_{1}\right)^{2}+\left(x_{2}-W_{2}\right)^{2}\right)+\frac{\imath}{2}\left(\dot{W}_{1} x_{1}+\dot{W}_{2} x_{2}\right)\right\}
$$

Here $A, B, W, W_{1}, W_{2}$ are arbitrary smooth functions on $t$.

Substituting the obtained expressions for the functions $Q, \omega_{1}, \omega_{2}$ into the last equation from the system (17) and splitting with respect to variables $x_{1}, x_{2}$ we get explicit forms of potentials $V\left(x_{1}, x_{2}\right)$ and systems of nonlinear $O D E s$ for unknown functions $A(t), B(t), W(t), W_{1}(t), W_{2}(t)$. We have succeeded in integrating these and in constructing all coordinate systems providing $S V$ in the initial equation (11).

Here we consider in detail integration of the fourth equation of system (7) for the case 2 from (8), since computations needed are not so lengthy as for other cases.

First, we make several important remarks which introduce an equivalence relation on the set of potentials $V\left(x_{1}, x_{2}\right)$.

Remark 1. The Schrödinger equation with the potential 


$$
V\left(x_{1}, x_{2}\right)=k_{1} x_{1}+k_{2} x_{2}+k_{3}+V_{1}\left(k_{2} x_{1}-k_{1} x_{2}\right),
$$

where $k_{1}, k_{2}, k_{3}$ are constants, is transformed to the Schrödinger equation with the potential

$$
V^{\prime}\left(x_{1}^{\prime}, x_{2}^{\prime}\right)=V_{1}\left(k_{2} x_{1}^{\prime}-k_{1} x_{2}^{\prime}\right)
$$

by the following change of variables:

$$
\begin{aligned}
t^{\prime} & =t, \quad \vec{x}^{\prime}=\vec{x}+t^{2} \vec{k}, \\
u^{\prime} & =u \exp \left\{\frac{\imath}{3}\left(k_{1}^{2}+k_{2}^{2}\right) t^{3}+\imath t\left(k_{1} x_{1}+k_{2} x_{2}\right)+\imath k_{3} t\right\} .
\end{aligned}
$$

It is readily seen that the class of Ansätze is transformed into itself by the above change of variables. That is why, potentials (9) and (10) are considered as equivalent.

Remark 2. The Schrödinger equation with the potential

$$
V\left(x_{1}, x_{2}\right)=k\left(x_{1}^{2}+x_{2}^{2}\right)+V_{1}\left(\frac{x_{1}}{x_{2}}\right)\left(x_{1}^{2}+x_{2}^{2}\right)^{-1}
$$

with $k=$ const is reduced to the Schrödinger equation with the potential

$$
V^{\prime}\left(x_{1}, x_{2}\right)=V_{1}\left(\frac{x_{1}^{\prime}}{x_{2}^{\prime}}\right)\left(x_{1}^{\prime 2}+x_{1}^{\prime 2}\right)^{-1}
$$

by the change of variables

$$
t^{\prime}=\alpha(t), \quad \vec{x}^{\prime}=\beta(t) \vec{x}, \quad u^{\prime}=u \exp \left\{\imath \gamma(t)\left(x_{1}^{2}+x_{2}^{2}\right)+\delta(t)\right\},
$$

where $(\alpha(t), \beta(t), \gamma(t), \delta(t))$ is an arbitrary solution of the system of ODEs

$$
\dot{\gamma}-4 \gamma^{2}=k, \quad \dot{\beta}-4 \gamma \beta=0, \quad \dot{\alpha}-\beta^{2}=0, \quad \dot{\delta}+4 \gamma=0
$$

such that $\beta \neq 0$.

Since the above change of variables does not alter the structure of the Ansatz (5), when classifying potentials $V\left(x_{1}, x_{2}\right)$ providing separability of the corresponding Schrödinger equation we consider potentials (12), (13) as equivalent.

Remark 3. It is well-known (see e.g. [11, 12]) that the general form of the invariance group admitted by Eq.(11) is as follows

$$
\begin{aligned}
& t^{\prime}=F(t, \vec{\theta}), \quad x_{a}^{\prime}=g_{a}(t, \vec{x}, \vec{\theta}), \quad a=1,2, \\
& u^{\prime}=h(t, \vec{x}, \vec{\theta}) u+U(t, \vec{x}),
\end{aligned}
$$

where $\vec{\theta}=\left(\theta_{1}, \theta_{2}, \ldots, \theta_{n}\right)$ are group parameters and $U(t, \vec{x})$ is an arbitrary solution of Eq.(11). 
The above transformations also do not alter the structure of the Ansatz (5). That is why, systems of coordinates $t^{\prime}, x_{1}^{\prime}, x_{2}^{\prime}$ and $t, x_{1}, x_{2}$ are considered as equivalent.

Now we turn to the integration of the fourth equation of system (7). Substituting into it the expressions for the functions $\omega_{1}, \omega_{2}, Q$ given by formulae 2 from (8) we get

$$
\begin{aligned}
V\left(x_{1}, x_{2}\right)= & \left(B_{01}\left(\omega_{1}\right)+B_{02}\left(\omega_{2}\right)\right) \exp \left\{-2\left(\omega_{1}-W\right)\right\}+\frac{1}{4}\left(\ddot{W}-\dot{W}^{2}\right) \\
& \times \exp \left\{2\left(\omega_{1}-W\right)\right\}+R_{0}(t)-\imath \dot{W} .
\end{aligned}
$$

In the above equality $B_{01}, B_{02}, R_{0}(t), W(t)$ are unknown functions to be determined from the requirement that the right-hand side of (14) does not depend on $t$.

Differentiating (14) with respect to $t$ and taking into account the equalities

$$
\omega_{1 t}=\dot{W}, \quad \omega_{2 t}=0
$$

we have

$$
\dot{W} \exp \left\{-2\left(\omega_{1}-W\right)\right\} \dot{B}_{01}+\dot{\alpha}(t) \exp \left\{2\left(\omega_{1}-W\right)\right\}+\dot{\beta}(t)=0,
$$

where $\alpha(t)=\frac{1}{4}\left(\ddot{W}-\dot{W}^{2}\right), \beta(t)=R_{0}-\imath \dot{W}$.

Cases $\dot{W}=0$ and $\dot{W} \neq 0$ have to be considered separately.

The case $1 . \dot{W}=0$.

In this case $W=C=$ const, $R_{0}=0$. Since coordinate systems $\omega_{1}, \omega_{2}$ and $\omega_{1}+$ $C_{1}, \omega_{2}+C_{2}$ are equivalent with arbitrary constants $C_{1}, C_{2}$, choosing $C_{1}=-C, C_{2}=0$ we can put $C=0$. Hence it immediately follows that

$$
V\left(x_{1}, x_{2}\right)=\left[B_{01}\left(\frac{1}{2} \ln \left(x_{1}^{2}+x_{2}^{2}\right)\right)+B_{02}\left(\arctan \frac{x_{1}}{x_{2}}\right)\right]\left(x_{1}^{2}+x_{2}^{2}\right)^{-1},
$$

where $B_{01}, B_{02}$ are arbitrary functions. And what is more, the Schrödinger equation (11) with such potential separates only in one coordinate system

$$
\omega_{1}=\frac{1}{2} \ln \left(x_{1}^{2}+x_{2}^{2}\right), \quad \omega_{2}=\arctan \frac{x_{1}}{x_{2}} .
$$

The case 2. $\dot{W} \neq 0$

Dividing (14) into $\dot{W} \exp \left\{-2\left(\omega_{1}-W\right)\right\}$ and differentiating the equality obtained with respect to $t$ we get

$$
\exp \left\{4 \omega_{1}\right\} \frac{d}{d t}\left(\dot{\alpha}(\dot{W})^{-1} \exp \{-4 W\}\right)+\exp \left\{2 \omega_{1}\right\} \frac{d}{d t}\left(\dot{\beta}(\dot{W})^{-1} \exp \{-2 W\}\right)=0
$$

whence

$$
\frac{d}{d t}\left(\dot{\alpha}(\dot{W})^{-1} \exp \{-4 W\}\right)=0, \quad \frac{d}{d t}\left(\dot{\beta}(\dot{W})^{-1} \exp \{-2 W\}\right)=0 .
$$

Integration of the above ODEs yields the following result: 


$$
\alpha=C_{1} \exp \{4 W\}+C_{2}, \quad \beta=C_{3} \exp \{2 W\}+C_{4},
$$

where $C_{\jmath}, \jmath=\overline{1,4}$ are arbitrary real constants.

Inserting the result obtained into (15) we get an equation for $B_{01}$

$$
\dot{B}_{01}=-4 C_{1} \exp \left\{4 \omega_{1}\right\}-2 C_{3} \exp \left\{2 \omega_{1}\right\},
$$

which general solution reads

$$
B_{01}=-C_{1} \exp \left\{4 \omega_{1}\right\}-C_{3} \exp \left\{2 \omega_{1}\right\}+C_{5} .
$$

In the above equality $C_{5}$ is an arbitrary real constant.

Substituting the expressions for $\alpha, \beta, B_{01}$ into Eq.(14) we have the explicit form of the potential $V\left(x_{1}, x_{2}\right)$

$$
V\left(x_{1}, x_{2}\right)=\left[B_{02}\left(\arctan \frac{x_{1}}{x_{2}}\right)+C_{5}\right]\left(x_{1}^{2}+x_{2}^{2}\right)^{-1}+C_{2}\left(x_{1}^{2}+x_{2}^{2}\right)+C_{4},
$$

where $B_{02}$ is an arbitrary function.

By force of the Remarks 1,2 we can choose $C_{2}=C_{4}=0$. Furthermore, due to arbitrariness of the function $B_{02}$ we can put $C_{5}=0$.

Thus, the case $\dot{W} \neq 0$ leads to the following potential:

$$
V\left(x_{1}, x_{2}\right)=B_{02}\left(\arctan \frac{x_{1}}{x_{2}}\right)\left(x_{1}^{2}+x_{2}^{2}\right)^{-1} .
$$

Substitution of the above expression into Eq.(14) yields second-order nonlinear $O D E$ for the function $W=W(t)$

$$
\ddot{W}-\dot{W}^{2}=4 C_{1} \exp \{4 W\},
$$

while the function $R_{0}$ is given by the formula

$$
R_{0}=\imath \dot{W}+C_{3} \exp \{2 W\}
$$

Integration of $O D E(18)$ is considered in detail in the Appendix 1. Its general solution has the form under $C_{1} \neq 0$

$$
W=-\frac{1}{2} \ln \left((a t-b)^{2}-4 C_{1}\right)+\frac{1}{2} \ln a
$$

under $C_{1}=0$

$$
W=a-\ln (t+b) .
$$

Substituting obtained expressions for $W$ into formulae 2 from (8) and taking into account the Remark 3 we arrive at the conclusion that the Schrödinger equation (11) 
with the potential (17) admits $S V$ in two coordinate systems. One of them is the polar coordinate system (16) and another one is the following:

$$
\omega_{1}=\frac{1}{2} \ln \left(x_{1}^{2}+x_{2}^{2}\right)-\frac{1}{2} \ln \left(t^{2} \pm 1\right), \quad \omega_{2}=\arctan \frac{x_{1}}{x_{2}} .
$$

Consequently, the case 2 from (8) gives rise to two classes of the separable Schrödinger equations (11).

Cases 1, 3, 4 from (8) are considered in an analogous way but computations involved are much more cumbersome. As a result, we obtain the following list of inequivalent potentials $V\left(x_{1}, x_{2}\right)$ providing separability of the Schrödinger equation.

1. $V\left(x_{1}, x_{2}\right)=V_{1}\left(x_{1}\right)+V_{2}\left(x_{2}\right)$;

(a) $V\left(x_{1}, x_{2}\right)=k_{1} x_{1}^{2}+k_{2} x_{1}^{-2}+V_{2}\left(x_{2}\right), \quad k_{2} \neq 0$;

i. $V\left(x_{1}, x_{2}\right)=k_{1} x_{1}^{2}+k_{2} x_{2}^{2}+k_{3} x_{1}^{-2}+k_{4} x_{2}^{-2}, \quad k_{3} k_{4} \neq 0$, $k_{1}^{2}+k_{2}^{2} \neq 0, k_{1} \neq k_{2}$

ii. $V\left(x_{1}, x_{2}\right)=k_{1} x_{1}^{2}+k_{2} x_{1}^{-2}, \quad k_{1} k_{2} \neq 0$;

iii. $V\left(x_{1}, x_{2}\right)=k_{1} x_{1}^{-2}+k_{2} x_{2}^{-2}$;

(b) $V\left(x_{1}, x_{2}\right)=k_{1} x_{1}^{2}+V_{2}\left(x_{2}\right)$;

i. $V\left(x_{1}, x_{2}\right)=k_{1} x_{1}^{2}+k_{2} x_{2}^{2}+k_{3} x_{2}^{-2}, \quad k_{1} k_{3} \neq 0, k_{1} \neq k_{2}$;

ii. $V\left(x_{1}, x_{2}\right)=k_{1} x_{1}^{2}+k_{2} x_{2}^{2}, \quad k_{1} k_{2} \neq 0, k_{1} \neq k_{2}$;

iii. $V\left(x_{1}, x_{2}\right)=k_{1} x_{1}^{2}+k_{2} x_{2}^{-2}, \quad k_{1} \neq 0$;

2. $V\left(x_{1}, x_{2}\right)=V_{1}\left(x_{1}^{2}+x_{2}^{2}\right)+V_{2}\left(\frac{x_{1}}{x_{2}}\right)\left(x_{1}^{2}+x_{2}^{2}\right)^{-1}$;

(a) $V\left(x_{1}, x_{2}\right)=V_{2}\left(\frac{x_{1}}{x_{2}}\right)\left(x_{1}^{2}+x_{2}^{2}\right)^{-1}$;

(b) $V\left(x_{1}, x_{2}\right)=k_{1}\left(x_{1}^{2}+x_{2}^{2}\right)^{-1 / 2}, \quad k_{1} \neq 0$;

3. $V\left(x_{1}, x_{2}\right)=\left(V_{1}\left(\omega_{1}\right)+V_{2}\left(\omega_{2}\right)\right)\left(\omega_{1}^{2}+\omega_{2}^{2}\right)^{-1}$, where $\omega_{1}^{2}-\omega_{2}^{2}=2 x_{1}, \omega_{1} \omega_{2}=x_{2}$;

4. $V\left(x_{1}, x_{2}\right)=\left(V_{1}\left(\omega_{1}\right)+V_{2}\left(\omega_{2}\right)\right)\left(\sinh ^{2} \omega_{1}+\sin ^{2} \omega_{2}\right)^{-1}$, where $\cosh \omega_{1} \cos \omega_{2}=x_{1}, \sinh \omega_{1} \sin \omega_{2}=x_{2}$;

5. $V\left(x_{1}, x_{2}\right)=0$. 
In the above formulae $V_{1}, V_{2}$ are arbitrary smooth functions, $k_{1}, k_{2}, k_{3}, k_{4}$ are arbitrary constants.

It should be emphasized that the above potentials are not inequivalent in a usual sense. These potentials differ from each other by the fact that the coordinate systems providing separability of the corresponding Schrödinger equations are different. As an illustration, we give the following figure:



$\underline{\text { Fig.1 }}$ 
where $r=\left(x_{1}^{2}+x_{2}^{2}\right)^{1 / 2}$ and by the symbol $V^{(\jmath)}, \jmath=1,4$ we denote the potential given in the above list under the number $\jmath$. Downarrows in the Fig. 1 indicate specifications of the potential $\left.V x_{1}, x_{2}\right)$ providing new possibilities to separate the corresponding Schrödinger equation (1).

The Schrödinger equation (11) with arbitrary function $V\left(x_{1}, x_{2}\right)$ (level 1 of the Fig.1) admits no separation of variables. Next, Eq.(1) with the "root" potentials $V^{(\jmath)}$ (level 2), $V_{1}, V_{2}$ being arbitrary smooth functions, separates in the Cartesian $(\jmath=1)$, polar $(\jmath=2)$, parabolic $(\jmath=3)$ and elliptic $(\jmath=4)$ coordinate systems, correspondingly. Specifying the functions $V_{1}, V_{2}$ (i.e. going down to the lower levels) new possibilities to separate variables in the Schrödinger equation (11) arise. For example, Eq.(1) with the potential $V_{2}\left(\frac{x_{1}}{x_{2}}\right) r^{-2}$, which is a particular case of the potential $V^{(2)}$, separates not only in the polar coordinate system (16) but also in the coordinate systems (19). The Schrödinger equation with the Coulomb potential $k_{1} r^{-1}$, which is a particular case of the potentials $V^{(2)}, V^{(3)}$, separates in two coordinate systems (namely, in the polar and parabolic coordinate systems, see below the Theorem 4). An another characteristic example is a transition from the potential $V^{(1)}$ to the potential $k_{1} x_{1}^{2}+V_{2}\left(x_{2}\right)$. The Schrödinger equation with the potential $V^{(1)}$ admits $S V$ in the Cartesian coordinate system $\omega_{0}=t, \omega_{1}=x_{1}, \omega_{2}=x_{2}$ only, while the one with the potential $k_{1} x_{1}^{2}+V_{2}\left(x_{2}\right)$ separates in seven $\left(k_{1}<0\right)$ or in three $\left(k_{1}>0\right)$ coordinate systems.

A complete list of coordinate systems providing $S V$ in the Schrödinger equations with the above given potentials takes two dozens of pages. Therefore, we restrict ourself to considering the Schrödinger equation with anisotropic harmonic oscillator potential $V\left(x_{1}, x_{2}\right)=k_{1} x_{1}^{2}+k_{2} x_{2}^{2}, k_{1} \neq k_{2}$ and Coulomb potential $V\left(x_{1}, x_{2}\right)=k_{1}\left(x_{1}^{2}+x_{2}^{2}\right)^{-1 / 2}$.

\section{Separation of variables in the Schrödinger equa- tion with the anisotropic harmonic oscillator and the Coulomb potentials.}

Here we will obtain all coordinate systems providing separability of the Schrödinger equation with the potential $V\left(x_{1}, x_{2}\right)=k_{1} x_{1}^{2}+k_{2} x_{2}^{2}$

$$
\imath u_{t}+u_{x_{1} x_{1}}+u_{x_{2} x_{2}}=\left(k_{1} x_{1}^{2}+k_{2} x_{2}^{2}\right) u .
$$

In the following, we consider the case $k_{1} \neq k_{2}$, because otherwise Eq.(1) is reduced to the free Schrödinger equation (see the Remark 2) which has been studied in detail in [1-3].

Explicit forms of the coordinate systems to be found depend essentially on the signs of the parameters $k_{1}, k_{2}$. We consider in detail the case, when $k_{1}<0, k_{2}>0$ (the cases $k_{1}>0, k_{2}>0$ and $k_{1}<0, k_{2}<0$ are handled in an analogous way). It means that Eq.(20) can be written in the form 


$$
\imath u_{t}+u_{x_{1} x_{1}}+u_{x_{2} x_{2}}+\frac{1}{4}\left(a^{2} x_{1}^{2}-b^{2} x_{2}^{2}\right) u=0,
$$

where $a, b$ are arbitrary non-null real constants (the factor $\frac{1}{4}$ is introduced for further convenience).

As stated above to describe all coordinate systems $t, \omega_{1}(t, \vec{x}), \omega_{2}(t, \vec{x})$ providing separability of Eq.(20) one has to construct the general solution of system (8) with $V\left(x_{1}, x_{2}\right)=-\frac{1}{4}\left(a^{2} x_{1}^{2}-b^{2} x_{2}^{2}\right)$. The general solution of equations 1-3 from (7) splits into four inequivalent classes listed in (8). Analysis shows that only solutions belonging to the first class can satisfy an equation 4 from (7).

Substituting the expressions for $\omega_{1}, \omega_{2}, Q$ given by the formulae 1 from (8) into the equation 4 from (17) with $V\left(x_{1}, x_{2}\right)=-\frac{1}{4}\left(a^{2} x_{1}^{2}-b^{2} x_{2}^{2}\right)$ and splitting with respect to $x_{1}, x_{2}$ one gets

$$
\begin{aligned}
& B_{01}\left(\omega_{1}\right)=\alpha_{1} \omega_{1}^{2}+\alpha_{2} \omega_{1}, B_{02}\left(\omega_{2}\right)=\beta_{1} \omega_{2}^{2}+\beta_{2} \omega_{2}, \\
& \left(\frac{\dot{A}}{A}\right)^{\cdot}-\left(\frac{\dot{A}}{A}\right)^{2}-4 \alpha_{1} A^{4}+a^{2}=0, \\
& \left(\frac{\dot{B}}{B}\right)^{\cdot}-\left(\frac{\dot{B}}{B}\right)^{2}-4 \beta_{1} B^{4}-b^{2}=0, \\
& \ddot{\theta}_{1}-2 \dot{\theta}_{1} \frac{\dot{A}}{A}-2\left(2 \alpha_{1} \theta_{1}+\alpha_{2}\right) A^{4}=0, \\
& \ddot{\theta}_{2}-2 \dot{\theta}_{2} \frac{\dot{B}}{B}-2\left(2 \beta_{1} \theta_{2}+\beta_{2}\right) B^{4}=0 .
\end{aligned}
$$

Here $\alpha_{1}, \alpha_{2}, \beta_{1}, \beta_{2}$ are arbitrary real constants.

Integration of the system of nonlinear ODEs (22)-(25) is carried out in the Appendix 1. Substitution of the formulae (A.2), (A.4)-(A.6), (A.8)-(A.11) into the corresponding expressions 1 from (8) yields a complete list of coordinate systems providing separability of the Schrödinger equation (21). These systems can be transformed to canonical form if we use the Remark 3.

The invariance group of Eq.(21) is generated by the following basis operators [11]:

$$
\begin{aligned}
P_{0} & =\partial_{t}, \quad I=u \partial_{u}, \quad M=\imath u \partial_{u}, \quad Q_{\infty}=U(t, \vec{x}) \partial_{u} \\
P_{1} & =\cosh a t \partial_{x_{1}}+\frac{\imath a}{2}\left(x_{1} \sinh a t\right) u \partial_{u}, \\
P_{2} & =\cos b t \partial_{x_{2}}-\frac{\imath b}{2}\left(x_{2} \sin b t\right) u \partial_{u}, \\
G_{1} & =\sinh a t \partial_{x_{1}}+\frac{\imath a}{2}\left(x_{1} \cosh a t\right) u \partial_{u}, \\
G_{2} & =\sin b t \partial_{x_{2}}+\frac{\imath b}{2}\left(x_{2} \cos b t\right) u \partial_{u},
\end{aligned}
$$


where $U(t, \vec{x})$ is an arbitrary solution of Eq. 21 .

Using the finite transformations generated by the infinitesimal operators (26) and the Remark 3 we can choose in the formulae (A.4)-(A.6), (A.8), (A.10), (A.11) $C_{3}=C_{4}=$ $D_{1}=0, D_{3}=D_{4}=0, C_{2}=D_{2}=1$. As a result, we come to the following assertion.

Theorem 1. The Schrödinger equation (21) admits SV in 21 inequivalent coordinate systems of the form

$$
\omega_{0}=t, \quad \omega_{1}=\omega_{1}(t, \vec{x}), \quad \omega_{2}=\omega_{2}(t, \vec{x}),
$$

where $\omega_{1}$ is given by one of the formulae from the first and $\omega_{2}$ - by one of the formulae from the second column of the Table 1.

Table 1.

\begin{tabular}{|c||c|}
\hline$\omega_{1}(t, \vec{x})$ & $\omega_{2}(t, \vec{x})$ \\
\hline$x_{1}(\sinh a(t+C))^{-1}+\alpha(\sinh a(t+C))^{-2}$ & $x_{2}(\sin b t)^{-1}+\beta(\sin b t)^{-2}$ \\
$x_{1}(\cosh a(t+C))^{-1}+\alpha(\cosh a(t+C))^{-2}$ & $x_{2}(\beta+\sin 2 b t)^{-1 / 2}$ \\
$x_{1} \exp ( \pm a t)+\alpha \exp ( \pm 4 a t)$ & $x_{2}$ \\
$x_{1}(\alpha+\sinh 2 a(t+C))^{-1 / 2}$ & \\
$x_{1}(\alpha+\cosh 2 a(t+C))^{-1 / 2}$ & \\
$x_{1}(\alpha+\exp ( \pm 2 a t))^{-1 / 2}$ & \\
$x_{1}$ & \\
\hline
\end{tabular}

Here $C, \alpha, \beta$ are arbitrary real constants.

There is no necessity to consider specially the case when in (20) $k_{1}>0, k_{2}<0$, since such an equation by the change of independent variables $u\left(t, x_{1}, x_{2}\right) \rightarrow u\left(t, x_{2}, x_{1}\right)$ is reduced to Eq.(21).

Below we adduce without proof the assertions describing coordinate systems providing $S V$ in Eq.(20) with $k_{1}<0, k_{2}<0$ and $k_{1}>0, k_{2}>0$.

Theorem 2. The Schrödinger equation

$$
\imath u_{t}+u_{x_{1} x_{1}}+u_{x_{2} x_{2}}+\frac{1}{4}\left(a^{2} x_{1}^{2}+b^{2} x_{2}^{2}\right) u=0
$$

with $a^{2} \neq 4 b^{2}$ admits $S V$ in 49 inequivalent coordinate systems of the form (27), where $\omega_{1}$ is given by one of the formulae from the first and $\omega_{2}-$ by one of the formulae from the second column of the Table 2. Provided $a^{2}=4 b^{2}$ one more coordinate system should be included into the above list, namely 


$$
\omega_{0}=t, \quad \omega_{1}^{2}-\omega_{2}^{2}=2 x_{1}, \quad \omega_{1} \omega_{2}=x_{2} .
$$

Table 2.

\begin{tabular}{|c||c|}
\hline$\omega_{1}(t, \vec{x})$ & $\omega_{2}(t, \vec{x})$ \\
\hline$x_{1}(\sinh a(t+C))^{-1}+\alpha(\sinh a(t+C))^{-2}$ & $x_{2}(\sinh b t)^{-1}+\beta(\sinh b t)^{-2}$ \\
$x_{1}(\cosh a(t+C))^{-1}+\alpha(\cosh a(t+C))^{-2}$ & $x_{2}(\cosh b t)^{-1}+\beta(\cosh b t)^{-2}$ \\
$x_{1} \exp ( \pm a t)+\alpha \exp ( \pm 4 a t)$ & $x_{2} \exp ( \pm b t)+\beta \exp ( \pm 4 b t)$ \\
$x_{1}(\alpha+\sinh 2 a(t+C))^{-1 / 2}$ & $x_{2}(\beta+\sinh 2 b t)^{-1 / 2}$ \\
$x_{1}(\alpha+\cosh 2 a(t+C))^{-1 / 2}$ & $x_{2}(\beta+\cosh 2 b t)^{-1 / 2}$ \\
$x_{1}(\alpha+\exp ( \pm 2 a t))^{-1 / 2}$ & $x_{2}(\beta+\exp ( \pm 2 b t))^{-1 / 2}$ \\
$x_{1}$ & $x_{2}$ \\
\hline
\end{tabular}

Here $C, \alpha, \beta$ are arbitrary constants.

Theorem 3. The Schrödinger equation

$$
\imath u_{t}+u_{x_{1} x_{1}}+u_{x_{2} x_{2}}-\frac{1}{4}\left(a^{2} x_{1}^{2}+b^{2} x_{2}^{2}\right) u=0
$$

with $a^{2} \neq 4 b^{2}$ admits $S V$ in 9 inequivalent coordinate systems of the form (2X), where $\omega_{1}$ is given by one of the formulae from the first and $\omega_{2}$-by one of the formulae from the second column of the Table 3. Provided $a^{2}=4 b^{2}$, the above list should be supplemented by the coordinate system (29).

Table 3.

\begin{tabular}{|c||c|}
\hline$\omega_{1}(t, \vec{x})$ & $\omega_{2}(t, \vec{x})$ \\
\hline$x_{1}(\sin a(t+C))^{-1}+\alpha(\sin a(t+C))^{-2}$ & $x_{2}(\sin b t)^{-1}+\beta(\sin b t)^{-2}$ \\
$x_{1}(\beta+\sin 2 a(t+C))^{-1 / 2}$ & $x_{2}(\beta+\sin 2 b t)^{-1 / 2}$ \\
$x_{1}$ & $x_{2}$ \\
\hline
\end{tabular}


Here $C, \alpha, \beta$ are arbitrary constants.

Remark 4. If we consider (1) as an equation for a complex-valued function $u$ of three complex variables $t, x_{1}, x_{2}$, then the cases considered in the Theorems 1-3 are equivalent. Really, replacing, when necessary, $a$ with $\imath a$ and $b$ by $\imath b$ we can always reduce Eqs.(21), (28) to the form (30). It means that coordinate systems presented in the Tables 1,2 are complex equivalent to those listed in the Table 3. But if $u$ is a complex-valued function of real variables $t, x_{1}, x_{2}$ it is not the case.

Theorem 4. The Schrödinger equation with the Coulomb potential

$$
\imath u_{t}+u_{x_{1} x_{1}}+u_{x_{2} x_{2}}-k_{1}\left(x_{1}^{2}+x_{2}^{2}\right)^{-1 / 2} u=0
$$

admits $S V$ in two coordinate systems (10), (29).

It is important to note that explicit forms of coordinate systems providing separability of Eqs.(21), (28), (30) depend essentially on the parameters $a, b$ contained in the potential $V\left(x_{1}, x_{2}\right)$. It means that the free Schrödinger equation $(V=0)$ does not admit $S V$ in such coordinate systems. Consequently, they are essentially new.

\section{Conclusion}

In the present paper we have studied the case when the Schrödinger equation (1) separates into one first-order and two second-order $O D E s$. It is not difficult to prove that there are no functions $Q(t, \vec{x}), \omega_{\mu}(t, \vec{x}), \mu=\overline{0,2}$ such that the Ansatz

$$
u=Q(t, \vec{x}) \varphi_{0}\left(\omega_{0}(t, \vec{x})\right) \varphi_{1}\left(\omega_{1}(t, \vec{x})\right) \varphi_{2}\left(\omega_{2}(t, \vec{x})\right)
$$

separates Eq.(11) into three second-order ODEs (see Appendix 2). Nevertheless, there exists a possibility for Eq.(11) to be separated into two first-order and one second-order $O D E s$ or into three first-order ODEs. This is a probable source of new potentials and new coordinate systems providing separability of the Schrödinger equation. It should be said that separation of the two-dimensional wave equation

$$
u_{t t}-u_{x x}=V(x) u
$$

into one first-order and one second-order $O D E s$ gives no new potentials as compared with separation of it into two second-order $O D E s$. But for some already known potentials new coordinate system providing separability of the above equation are obtained [9].

Let us briefly analyze a connection between separability of Eq.(1) and its symmetry properties. It is well-known that each solution of the free Schrödinger equation with separated variables is a common eigenfunction of two mutually commuting second-order symmetry operators of the said equation [2, 3]. And what is more, separation constants $\lambda_{1}, \lambda_{2}$ are eigenvalues of these symmetry operators.

We will establish that the same assertion holds for the Schrödinger equation (11). Let us make in Eq.(1) the following change of variables: 


$$
u=Q(t, \vec{x}) U\left(t, \omega_{1}(t, \vec{x}), \omega_{2}(t, \vec{x})\right),
$$

where $\left(Q, \omega_{1}, \omega_{2}\right)$ is an arbitrary solution of the system of PDEs (7).

Substituting the expression (31) into (11) and taking into account equations (7) we get

$$
Q\left(\imath U_{t}+\left(U_{\omega_{1} \omega_{1}}-B_{01}\left(\omega_{1}\right) U\right) \omega_{1 x_{a}} \omega_{1 x_{a}}+\left(U_{\omega_{2} \omega_{2}}-B_{02}\left(\omega_{2}\right) U\right) \omega_{2 x_{a}} \omega_{2 x_{a}}\right)=0
$$

Resolving equations 2 from the system (7) with respect to $\omega_{1 x_{a}} \omega_{1 x_{a}}$ and $\omega_{2 x_{a}} \omega_{2 x_{a}}$ we have

$$
\begin{aligned}
\omega_{1 x_{a}} \omega_{1 x_{a}} & =\frac{1}{\delta}\left(R_{2}(t) B_{21}\left(\omega_{2}\right)-R_{1}(t) B_{22}\left(\omega_{2}\right)\right), \\
\omega_{2 x_{a}} \omega_{2 x_{a}} & =\frac{1}{\delta}\left(R_{1}(t) B_{12}\left(\omega_{1}\right)-R_{2}(t) B_{11}\left(\omega_{1}\right)\right) .
\end{aligned}
$$

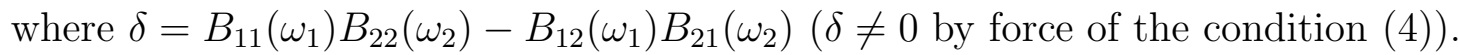

Substitution of the above equalities into Eq.(32) with subsequent division by $Q \neq 0$ yields the following $P D E$ :

$$
\begin{aligned}
\imath U_{t} & +\frac{R_{1}(t)}{\delta}\left(B_{12}\left(\omega_{1}\right)\left(U_{\omega_{2} \omega_{2}}-B_{02}\left(\omega_{2}\right) U\right)-B_{22}\left(\omega_{2}\right)\left(U_{\omega_{1} \omega_{1}}-B_{01}\left(\omega_{1}\right) U\right)\right) \\
& +\frac{R_{2}(t)}{\delta}\left(B_{21}\left(\omega_{2}\right)\left(U_{\omega_{1} \omega_{1}}-B_{01}\left(\omega_{1}\right) U\right)-B_{11}\left(\omega_{1}\right)\left(U_{\omega_{2} \omega_{2}}-B_{02}\left(\omega_{2}\right) U\right)\right)=0 .
\end{aligned}
$$

Thus, in the new coordinates $t, \omega_{1}, \omega_{2}, U\left(t, \omega_{1}, \omega_{2}\right)$ Eq.(1) takes the form (33).

By direct (and very cumbersome) computation one can check that the following second-order differential operators:

$$
\begin{aligned}
& X_{1}=\frac{B_{22}\left(\omega_{2}\right)}{\delta}\left(\partial_{\omega_{1}}^{2}-B_{01}\left(\omega_{1}\right)\right)-\frac{B_{12}\left(\omega_{1}\right)}{\delta}\left(\partial_{\omega_{2}}^{2}-B_{02}\left(\omega_{2}\right)\right), \\
& X_{2}=-\frac{B_{21}\left(\omega_{2}\right)}{\delta}\left(\partial_{\omega_{1}}^{2}-B_{01}\left(\omega_{1}\right)\right)+\frac{B_{11}\left(\omega_{1}\right)}{\delta}\left(\partial_{\omega_{2}}^{2}-B_{02}\left(\omega_{2}\right)\right),
\end{aligned}
$$

commute under arbitrary $B_{0 a}, B_{a b}, a, b=1,2$, i.e.

$$
\left[X_{1}, X_{2}\right] \equiv X_{1} X_{2}-X_{2} X_{1}=0 \text {. }
$$

After being rewritten in terms of the operators $X_{1}, X_{2}$ Eq.(33) reads

$$
\left(\imath \partial_{t}-R_{1}(t) X_{1}-R_{2}(t) X_{2}\right) U=0
$$

Since the relations 


$$
\left[\imath \partial_{t}-R_{1}(t) X_{1}-R_{2}(t) X_{2}, X_{a}\right]=0, a=1,2
$$

hold, operators $X_{1}, X_{2}$ are mutually commuting symmetry operators of Eq.([33). Furthermore, solution of Eq.(33) with separated variables $U=\varphi_{0}(t) \varphi_{1}\left(\omega_{1}\right) \varphi_{2}\left(\omega_{2}\right)$ satisfies the identities

$$
X_{a} U=\lambda_{a} U, a=1,2 .
$$

Consequently, if we designate by $X_{1}^{\prime}, X_{2}^{\prime}$ the operators $X_{1}, X_{2}$ written in the initial variables $t, \vec{x}, u$, then we get from (34)-(36) the following equalities:

$$
\begin{aligned}
& {\left[\imath \partial_{t}+\triangle-V\left(x_{1}, x_{2}\right), X_{a}^{\prime}\right]=0, a=1,2,} \\
& {\left[X_{1}^{\prime}, X_{2}^{\prime}\right]=0,} \\
& X_{a}^{\prime} u=\lambda_{a} u, a=1,2 .
\end{aligned}
$$

where $u=Q(t, \vec{x}) \varphi_{0}(t) \varphi_{1}\left(\omega_{1}\right) \varphi_{2}\left(\omega_{2}\right)$.

It means that each solution with separated variables is a common eigenfunction of two mutually commuting symmetry operators $X_{1}^{\prime}, X_{2}^{\prime}$ of the Schrödinger equation (1), separation constants $\lambda_{1}, \lambda_{2}$ being their eigenvalues.

Detailed study of the said operators as well as analysis of separated ODEs for functions $\varphi_{\mu}, \mu=\overline{0,2}$ (in the way as it is done for the free Schrödinger equation in [2, 3]) is in progress and will be a topic of our future publications.

\section{Acknowledgments}

When the paper was in the last stage of preparation, one of the authors (R.Zhdanov) was supported by the Alexander von Humboldt Foundation. Taking an opportunity he wants to express his gratitude to Director of Arnold Sommerfeld Institute for Mathematical Physics Professor H.-D.Doebner for hospitality.

\section{Appendix 1. Integration of nonlinear ODEs (22)-(25).}

Evidently, equations (22)-(25) can be rewritten in the following unified form:

$$
\left(\frac{\dot{y}}{y}\right)^{\cdot}-\left(\frac{\dot{y}}{y}\right)^{2}-4 \alpha y^{4}=k, \quad \ddot{z}-2 \dot{z} \frac{\dot{y}}{y}-2(2 \alpha z+\beta) y^{4}=0 .
$$

Provided $k=-a^{2}<0$, system (A.1) coincides with equations (22), (24) and under $k=b^{2}>0-$ with equations (23), (25).

First of all, we note that the function $z=z(t)$ is determined up to addition of an arbitrary constant. Really, the coordinate functions $\omega_{a}$ have the following structure: 


$$
\omega_{a}=y x_{a}+z, a=1,2 .
$$

But the coordinate system $t, \omega_{1}, \omega_{2}$ is equivalent to the coordinate system $t, \omega_{1}+$ $C_{1}, \omega_{2}+C_{2}, C_{a} \in R^{1}$. Hence it follows that the function $z(t)$ is equivalent to the function $z(t)+C$ with arbitrary real constant $C$. Consequently, provided $\alpha \neq 0$, we can choose in (A.1) $\beta=0$.

The case 1. $\alpha=0$.

On making in (A.1) the change of variables

$$
w=\frac{\dot{y}}{y}, \quad v=\frac{z}{y}
$$

we get

$$
\dot{w}=w^{2}+k, \quad \ddot{v}+k v=2 \beta y^{3} .
$$

First, we consider the case $k=-a^{2}<0$. Then the general solution of the first equation from (A.3) is given by one of the formulae

$$
w=-a \operatorname{coth} a\left(t+C_{1}\right), \quad w=-a \tanh a\left(t+C_{1}\right), \quad w= \pm a, \quad C_{1} \in R^{1},
$$

whence

$$
y=C_{2} \sinh ^{-1} a\left(t+C_{1}\right), \quad y=C_{2} \cosh ^{-1} a\left(t+C_{1}\right), \quad y=C_{2} \exp ( \pm a t), C_{2} \in R^{1} .
$$

The second equation of system (A.3) is a linear inhomogeneous ODE. Its general solution after being substituted into (A.2) yields the following expression for $z(t)$ :

$$
\begin{aligned}
& \left(C_{3} \cosh a t+C_{4} \sinh a t\right) \sinh ^{-1} a\left(t+C_{1}\right)+\frac{\beta C_{2}^{4}}{a^{2}} \sinh ^{-2} a\left(t+C_{1}\right), \\
& \left(C_{3} \cosh a t+C_{4} \sinh a t\right) \cosh ^{-1} a\left(t+C_{1}\right)+\frac{\beta C_{2}^{4}}{a^{2}} \cosh ^{-2} a\left(t+C_{1}\right), \\
& \left(C_{3} \cosh a t+C_{4} \sinh a t\right) \exp ( \pm a t)+\frac{\beta C_{2}^{4}}{4 a^{2}} \exp ( \pm 4 a t), \quad C_{3}, C_{4} \subset R^{1} .
\end{aligned}
$$

The case $k=b^{2}>0$ is treated in an analogous way, the general solution of (A.1) being given by the formulae

$$
\begin{aligned}
& y=D_{2} \sin ^{-1} b\left(t+D_{1}\right), \\
& z=\left(D_{3} \cos b t+D_{4} \sin b t\right) \sin ^{-1} b\left(t+D_{1}\right)+\frac{\beta D_{2}^{4}}{b^{2}} \sin ^{-2} b\left(t+D_{1}\right),
\end{aligned}
$$

where $D_{1}, D_{2}, D_{3}, D_{4}$ are arbitrary real constants.

The case 2. $\alpha \neq 0, \beta=0$.

On making in (A.1) the change of variables 


$$
y=\exp w, \quad v=\frac{z}{y}
$$

we have

$$
\ddot{w}-\dot{w}^{2}=k+\alpha \exp 4 w, \quad \ddot{v}+k v=0 .
$$

The first $O D E$ from (A.7) is reduced to the first-order linear $O D E$

$$
\frac{1}{2} \frac{d p(w)}{d w}-p(w)=k+\alpha \exp 4 w
$$

by the substitution $\dot{w}=(p(w))^{1 / 2}$, whence

$$
p(w)=\alpha \exp 4 w+\gamma \exp 2 w-k, \gamma \in R^{1} .
$$

Equation $\dot{w}=(p(w))^{1 / 2}$ has a singular solution $w=C=$ const such that $p(C)=0$. If $\dot{w} \neq 0$, then integrating the equation $\dot{w}=p(w)$ and returning to the initial variable $y$ we get

$$
\int^{y(t)} \frac{d \tau}{\tau\left(\alpha \tau^{4}+\gamma \tau^{2}-k\right)^{1 / 2}}=t+C_{1} .
$$

Taking the integral in the left-hand side of the above equality we obtain the general solution of the first $O D E$ from (A.1). It is given by the following formulae:

$\underline{\text { under } k=-a^{2}<0}$

$$
\begin{aligned}
& y=C_{2}\left(\alpha+\sinh 2 a\left(t+C_{1}\right)\right)^{-1 / 2}, \\
& y=C_{2}\left(\alpha+\cosh 2 a\left(t+C_{1}\right)\right)^{-1 / 2}, \\
& y=C_{2}(\alpha+\exp ( \pm 2 a t))^{-1 / 2},
\end{aligned}
$$

$\underline{\text { under } k=b^{2}>0}$

$$
y=D_{2}\left(\alpha+\sin 2 b\left(t+D_{1}\right)\right)^{-1 / 2} .
$$

Here $C_{1}, C_{2}, D_{1}, D_{2}$ are arbitrary real constants.

Integrating the second $O D E$ from (A.7) and returning to the initial variable $z$ we have $\underline{\text { under } k=-a^{2}<0}$

$$
z=y(t)\left(C_{3} \cosh a t+C_{4} \sinh a t\right)
$$

$\underline{\text { under } k=b^{2}>0}$

$$
z=y(t)\left(D_{3} \cos b t+D_{4} \sin b t\right),
$$

where $C_{3}, C_{4}, D_{3}, D_{4}$ are arbitrary real constants. 
Thus, we have constructed the general solution of the system of nonlinear ODEs (A.1) which is given by the formulae (A.5)-(A.11).

\section{Appendix 2. Separation of Eq.(1) into three second-order ODEs.}

Suppose that there exists an Ansatz

$$
u=Q(t, \vec{x}) \varphi_{0}\left(\omega_{0}(t, \vec{x})\right) \varphi_{1}\left(\omega_{1}(t, \vec{x})\right) \varphi_{2}\left(\omega_{2}(t, \vec{x})\right)
$$

which separates the Schrödinger equation into three second-order ODEs

$$
\begin{aligned}
& \frac{d^{2} \varphi_{0}}{d \omega_{0}^{2}}=U_{0}\left(\omega_{0}, \varphi_{0}, \frac{d \varphi_{0}}{d \omega_{0}} ; \lambda_{1}, \lambda_{2}\right), \\
& \frac{d^{2} \varphi_{1}}{d \omega_{1}^{2}}=U_{1}\left(\omega_{1}, \varphi_{1}, \frac{d \varphi_{1}}{d \omega_{1}} ; \lambda_{1}, \lambda_{2}\right), \\
& \frac{d^{2} \varphi_{2}}{d \omega_{2}^{2}}=U_{2}\left(\omega_{2}, \varphi_{2}, \frac{d \varphi_{2}}{d \omega_{2}} ; \lambda_{1}, \lambda_{2}\right)
\end{aligned}
$$

according to the Definition 1.

Substituting the Ansatz (A.12) into Eq.(11) and excluding the second derivatives $\frac{d^{2} \varphi_{\mu}}{d \omega_{\mu}^{2}}$, $\mu=\overline{0,2}$ according to Eqs.(A.13) we get

$$
\begin{aligned}
& \imath\left(Q_{t} \varphi_{0} \varphi_{1} \varphi_{2}+Q \omega_{0 t} \dot{\varphi}_{0} \varphi_{1} \varphi_{2}+Q \omega_{1 t} \varphi_{0} \dot{\varphi}_{1} \varphi_{2}+Q \omega_{2 t} \varphi_{0} \varphi_{1} \dot{\varphi}_{2}\right)+(\triangle Q) \varphi_{0} \varphi_{1} \varphi_{2} \\
& \quad+2 Q_{x_{a}} \omega_{0 x_{a}} \dot{\varphi}_{0} \varphi_{1} \varphi_{2}+2 Q_{x_{a}} \omega_{1 x_{a}} \varphi_{0} \dot{\varphi}_{1} \varphi_{2}+2 Q_{x_{a}} \omega_{2 x_{a}} \varphi_{0} \varphi_{1} \dot{\varphi}_{2}+Q\left(\left(\triangle \omega_{0}\right) \dot{\varphi}_{0} \varphi_{1} \varphi_{2}\right. \\
& \quad+\left(\triangle \omega_{1}\right) \varphi_{0} \dot{\varphi}_{1} \varphi_{2}+\left(\triangle \omega_{2}\right) \varphi_{0} \varphi_{1} \dot{\varphi}_{2}+\omega_{0 x_{a}} \omega_{0 x_{a}} U_{0} \varphi_{1} \varphi_{2}+\omega_{1 x_{a}} \omega_{1 x_{a}} \varphi_{0} U_{1} \varphi_{2} \\
& \left.\quad+\omega_{2 x_{a}} \omega_{2 x_{a}} \varphi_{0} \varphi_{1} U_{2}+2 \omega_{0 x_{a}} \omega_{1 x_{a}} \dot{\varphi}_{0} \dot{\varphi}_{1} \varphi_{2}+2 \omega_{0 x_{a}} \omega_{2 x_{a}} \dot{\varphi}_{0} \varphi_{1} \dot{\varphi}_{2}+2 \omega_{1 x_{a}} \omega_{2 x_{a}} \varphi_{0} \dot{\varphi}_{1} \dot{\varphi}_{2}\right) \\
& \quad=V Q \varphi_{0} \varphi_{1} \varphi_{2} .
\end{aligned}
$$

Splitting the above equality with respect to $\dot{\varphi}_{0} \dot{\varphi}_{1}, \dot{\varphi}_{0} \dot{\varphi}_{2}, \dot{\varphi}_{1} \dot{\varphi}_{2}$ we obtain the equalities:

$$
\omega_{0 x_{a}} \omega_{1 x_{a}}=0, \quad \omega_{0 x_{a}} \omega_{2 x_{a}}=0, \quad \omega_{1 x_{a}} \omega_{2 x_{a}}=0 .
$$

Since the functions $\omega_{\mu}, \mu=\overline{0,2}$ are real-valued, equalities (A.14) mean that there are three real two-component vectors which are mutually orthogonal. This is possible only if one of them is a null-vector. Without loss of generality we may suppose that $\left(\omega_{0 x_{1}}, \omega_{0 x_{2}}\right)=(0,0)$, whence $\omega_{0}=f(t) \sim t$.

Consequently, Ansatz (A.12) necessarily takes the form (5). But Ansatz (5) can not separate Eq.(1) into three second-order $O D E s$, since it contains no second-order derivative with respect to $t$.

Thus, we have proved that the Schrödinger equation (1) is not separable into three second-order ODEs. 


\section{References}

[1] C.Boyer, SIAM J.Math.Anal. 7, 230 (1976).

[2] C.Boyer, E.Kalnins and W.Miller, J.Math.Phys. 16, 499 (1975).

[3] W.Miller, Symmetry and Separation of Variables (Addison-Wesley, Massachusetts, 1977).

[4] V.N.Shapovalov and N.B.Sukhomlin, Izvestiya Vuzov,Fizika N 12, 268 (1974).

[5] A.G.Nikitin, S.P.Onufriychuk and W.I.Fushchych, Teoret.Matem.Fizika 12, 268 (1992).

[6] W.I.Fushchych, R.Z.Zhdanov and I.V.Revenko, Proc.Ukrain.Acad.Sci. N 1, 27 (1993).

[7] W.I.Fushchych, in: Symmetry in Mathematical Physics Problems (Inst.of Math., Kiev, 1981), p.6.

[8] T.H.Koornwinder, Lect.Notes in Math. 810, 240 (1980).

[9] R.Z.Zhdanov, I.V.Revenko and W.I.Fushchych, J.Phys.A: Math.Gen. 26, 5959 (1993).

[10] R.Z.Zhdanov, J.Phys.A: Math.Gen. 27, L291 (1994).

[11] C.Boyer Helv.Phys.Acta 47, 589 (1974).

[12] U.Niederer Helv.Phys.Acta 45, 802 (1972). 